\title{
Macroscopic Magnetic Islands and Plasma Energy Transport
}

\author{
F. Porcelli \\ E. Rossi \\ G. Cima \\ A. Wootton \\ This paper was prepared for submittal to the \\ Workshop on Fusion Related Physics and \\ Engineering in Small Devices \\ Trieste, Italy \\ October 6-8, 1998
}

December 3, 1998

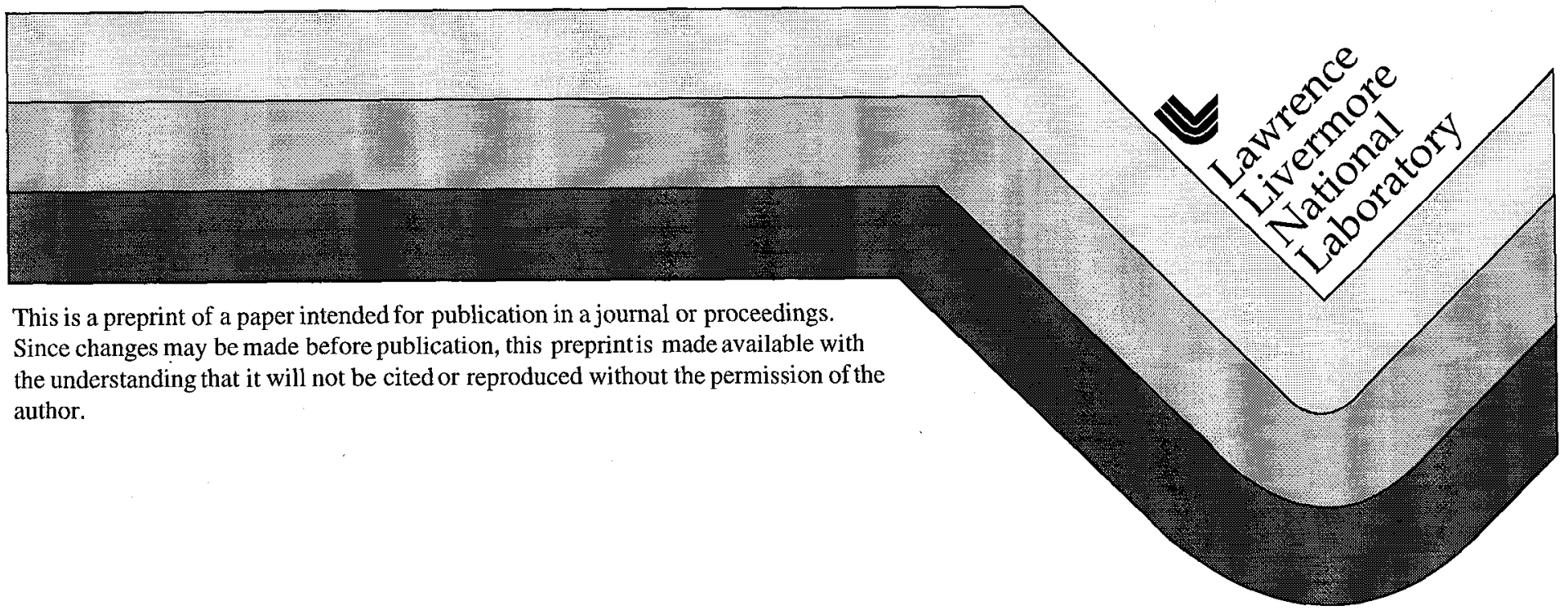




\section{DISCLAIMER}

This document was prepared as an account of work sponsored by an agency of the United States Government. Neither the United States Government nor the University of California nor any of their employees, makes any warranty, express or implied, or assumes any legal liability or responsibility for the accuracy, completeness, or usefulness of any information, apparatus, product, or process disclosed, or represents that its use would not infringe privately owned rights. Reference herein to any specific commercial product, process, or service by trade name, trademark, manufacturer, or otherwise, does not necessarily constitute or imply its endorsement, recommendation, or favoring by the United States Government or the University of California. The views and opinions of authors expressed herein do not necessarily state or reflect those of the United States Government or the University of California, and shall not be used for advertising or product endorsement purposes. 


\title{
Macroscopic magnetic islands and plasma energy transport
}

\author{
F. Porcelli ${ }^{1}$, E. Rossi ${ }^{1}$, G. Cima ${ }^{2}$, A. Wootton ${ }^{3}$, LLNL \\ Istituto Nazionale Fisica della Materia, Department of Energetics, \\ Politecnico di Torino, Itlay, ${ }^{2}$ Fusion Research Center, University of Texas at \\ Austin, U.S.A. \\ ${ }^{3}$ Lawrence Livermore National Laboratory
}

\begin{abstract}
A model is presented, based on the combined effects of $m=n=1$ magnetic island dynamics, localized heat sources, large heat diffusivity along magnetic field lines and plasma rotation, which may explain the multipeaked temperature profiles and transport barriers observed in tokamak plasmas heated by electron cyclotron resonant waves.
\end{abstract}

Experiments in magnetically confined, high temperature plasmas heated by intense electromagnetic waves at the electron cyclotron resonance frequency, have revealed peculiar and hitherto unexplained plasma behavior. The most striking results [1], come from the Rijnhuizen Tokamak Project (RTP) where electron cyclotron heating (ECH) with maximum power $\mathrm{P} \approx 360 \mathrm{k} \mathrm{W}$ is applied to the toroidal plasma. In this experiment, a double-pulse, multiposition Thomson scattering diagnostic measures the electron temperature profile with a very high spatial resolution. In the plasma central region, led the TRP group to suggest a "filamentary" structure. In addition ECH experiments in TRP [2] have revealed very sharp temperature gradients just outside the sawtooth mixing radius. Other minor structures in the $\mathrm{T}_{e}$ profiles are sometimes observed near rational surfaces with $q>1$. The sharp gradients at the sawtooth mixing radius, as well as the other minor structures, have been interpreted as due to the presence of "transport barriers" on rational $q$ surfaces $+[1]-[3]$. 
The RTP results have rased itie question whether the observed pectliar behavior is specific to $E C H$; ot whether is to fact universal to all tokamak plasmas, but can be recognized only with a sophisticated temperature diag nostics Clearly, an answer to this question relies on out understanding of the underlying magnetic structure. It must be emphasized that this structure in the plasma central region is strongly affected by the so-called sawtooth relaxation oscillations [4], associated with the instability of a resistive kink mode with toroidal $n=1$ and dominant poloidal $\mathrm{m}=1$ mode numbers, which results in a periodically growing $n=m=1$ magnetic island.

Pecularities in plasma behavior in the presence of ECF have been reported in other tokamaks as well. In this Letter, we shall concentrate on results from the Texas Experimental Tokaxak Upgrade (TEXT-UT) [5]. These results, together with nonstandard sawtooth traces observed in other ECH tokamak experiments (see; e.g.; Ref. $[6]$ and other references therein); strongly suggest that the peculiar behavior is the outcome of arr interplay between sawteeth and EOH. The objective of this Letter is to clarify this interplay from a theoretical standpoint. We ptesent a model for the evolution of the electron temperature profiles, based on the following essential ingrcdients: (i) a reconstruction of magnetic surfaces based on the sawtooth recontuection process;: (ii) the presence of a localized electron heat source; (iii) the effect of plasma rotation; (iv) the effect of arisotiopic heat diffusion, whereby the paraflel (along the field lines) heat diffusion coefficient, $X$ ll, is taken as very large, and the perperidieular coefficient, $\chi_{\perp}$, as relatively small, on the sawtooth period time scale and spatial extent. This model is able to reproduce quatitatively the observed features of the TEXT-U and RTP temperature profiles, in particulax sharp gradients at the sawtooth mixing radius and several peaks, even when a strictly constant $\chi \perp$ profile is assumed What is special with ECH, in contrast with other heating schemes, is that electron cyclotinn waves transfer their energy directly to the electrons, witl no delay. and in a very localized deposition region [7]. This heat is then transported very rapidy along field lincs to electrons in other parts of the plasma because of very large parallel heat conduction and plasma totation. These features are fully exploited in our rnodel.

Let us first summarice the relevant restlts from TEXT-U. [5]. The main tokamak parameters are $\mathrm{R}=1.05 \mathrm{mi}, \ddot{\mathrm{a}}=0.27 \mathrm{~m}, B \approx 2 \mathrm{~T}, I_{\mathrm{p}} \approx 230 \mathrm{kA}$ and central electron density $n_{\mathrm{e}} \approx 3.0 \times 10^{19} \mathrm{~m}^{-3}$. The experiment is equipped with an ECH system which delivers a maximum power $P \approx 270 \mathrm{~kW}$ : The electron 
temperature is measured by an Electron Cyclotron Emission (ECE) diagnostic. The typical spatial resolution of the ECE signal is $O(1 \mathrm{~cm})$, which is rot as good as the Thompson scattering system on RTP. On the other hand, the ECE diagnostic on 'TEXY-U has a very high temporal resolution, $O(20 \mu)$, over an extended time interval. In this sense, the temperature measurements on TEXT-U can be considered as complementary to those on RTP.
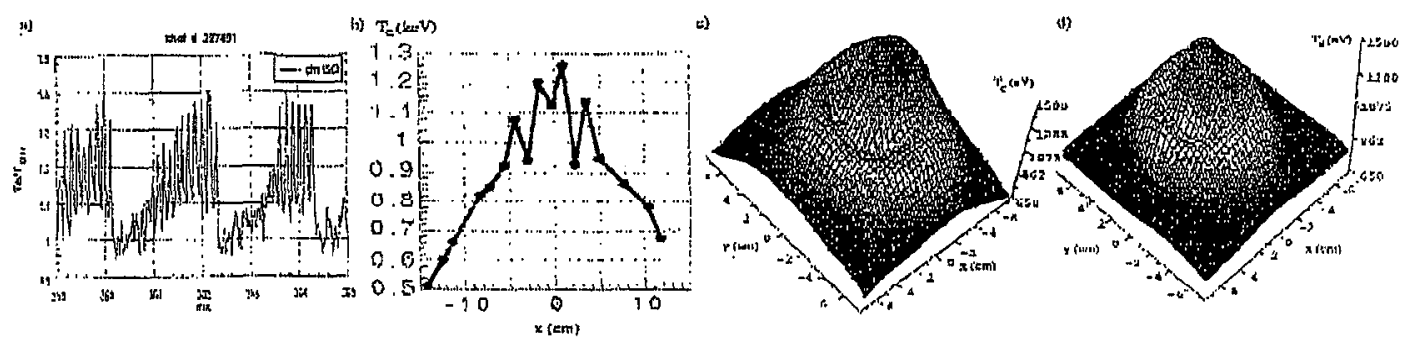

Figure 1: Electron temperature in TEXT U. (a): central $T_{0}$ evolution; (b): an example of $T_{e}$ profiles; $(c)$ and (d): $3-D$ reconstructions.

Figure 1 shows an example of the central electron temperature evolution in TEXT-U. Shown in figure 1(a) are nearly three sawtooth ramps, with a period $\tau_{\text {saw }} \approx 2 \mathrm{~ms}$, and with fast oscillations superimposed. The period of the fast oscillations matches the plasma toroidal rotation period, $\tau_{\text {rot }} \approx 160 \mu$ in this case. If these oscillations are indeed due to the presence of an $m=1$, $n=1$ magnetic island, then this island is formed early on duxing each sawtooth ramp and maintains a nearly saturated amplitude for most of the ramp. These ramps look different from Ohmic sawtooth ramps in TEXT-U, which exhibit detectable MHD Huctuations only near the tops. This suggests that the auxiliary heating influences $m=n=1$ mode stability, perhaps indirectly through the production of peaked pressure profiles. Fig. 1(b) presents an example of an ECE $T_{e}$ profile; a sequence of these profiles can be found in Fig. 10 of Ref. [5]. The profiles at time intervals $t_{2}-t_{I} \approx \tau_{\text {rot }}$ nearly coincide, which confirms that we are indeed observing a toroidal $n=1$ structure. These profiles exhibit four to five peaks and are similar to the filament-like temper. ature structures previously reported in RTP. However, the RTP observations lack time history. With the temporal and spatial resolution available on TEXT-U, we find that the structures are cyclic and consistent with a fixed structure in the rotating plasma frame. The main poloidal $m=1$ structure 
car be deduced from 3-D reconstructions of the type shown in Figs. $1(\mathrm{c})$ (d); where the time variable has been transformed into the poloidal angle variable under the assumption of rigid rotationi

Let tis now present our theoretical model. We assume, for simplicity, a "rectified" tokamak, i.e a cylindrical plasma column with periodic boundary conditions at the cylinder bases $(z=0$ and $z=L)$. In the absence of an $m=1$ island, mágnetic surface coss sections are concentric circles of constant normalized helical flux, $\psi_{* i n}(r)=\int_{0}^{r^{2}}\left[g_{i n}(x)^{-7}-1\right] d x$, where the subscript "in" stands" for "initial", i.e before the island formation ror a typical (i.e. monotomic) $q_{i n}(r)$ profile, $\psi_{* i z}(r)$ has a maximum at the $\dot{q}_{i n}=1$ radius, $r=r_{s}$. The growth of an m=1 island is "brought about by a rigid shift of sutfaces within the original $q_{i n}=1$ surface. The helical flux, $\psi_{*}(r, \alpha ; t)$, where $\alpha=\theta-2 \pi z / I$, remains nearly constart on moving fuid elexterits. At each stage of the reconnection process, the island separatrix is formed by two circles of radii $r_{1 s_{p}}<r_{s}$ and $T_{2 s p}>r_{s}$, where $\psi_{-i n}\left(r_{1 s p}\right)=\psi_{-i n}\left(r_{z s p}\right)$, with the center of the inter circle (i.e. the original magnetic axis) displeced from its initial position by a distance $\xi(t)=r_{2 s p}-r_{\text {tsp }}$. In addition, toroidal flux conservation is assumed; with $B_{z} \approx$ const; this is equivalent to area conservation, i.e. when the island separatrix later evolves into the croissant-shaped stuface, the area encircled in the poloidal plane by the surface is constarit and equal to $A=\pi\left(r_{2 s p}^{2}-r_{1 s p}^{2}\right)$ The contour of the croissant is also a surface of constant helical Alux, specifically $\psi_{*}\left(r_{2} \alpha ; \xi\right)=\psi_{* i n}\left(r_{1 s p}\right)$. This model for the $m=n=1$ island evolution is based on the specific convection pattern associated with the $m=n=1$ resistive internal kink roode [8] and on the ideal MHD assumption of frozen maginetic flux through moving furid elements, approximately valid everywhere except in the ixmediate neighborhood of the islarid X-point and current sheet:

The well known Kadontsev model [9] for the sawtooth Telaxation cot responds to the two basic reconrection rules described above and to the following assertions: (i) the teconinection process proceeds until full reconrection, i.e. until $\xi(t)$ attains its maximum value, $r_{m i x}$; called mixing radius and defined by $\psi_{* i n}\left(r_{m i x}\right)=\psi_{* i n}(0)$; when $\xi=r_{m i x}$, poloidal symmetry is restored; the island 0 -point having evolved into the new thagnetic axis, while the island X-point and the original axis annihilate each other; (ii) the time Tor $\xi$ to evolve from $\xi=0$ to $\xi=\tau_{\text {initi }}$ is $\tau_{K} \approx\left(\tau_{R} \tau_{A}\right)^{1 / 2}$, with $\tau_{A}$ a typical Alfvén time and $\tau_{r}$ the global resistive diffusion time In our model, we as sume that the island growth initially follows the two basic rules of the helical 
flux and area conservation, but does not necessarily evolve to full reconnection. In addition, we do not attempt to describe the dynamical evolution; rather, the function $\xi(t)$ is inferred from the experiments. We observe, however, that $\tau_{K}=O(1 \mathrm{~ms})$ for TEXT-U parameters, in fair agreement with the experimental sawtooth period.

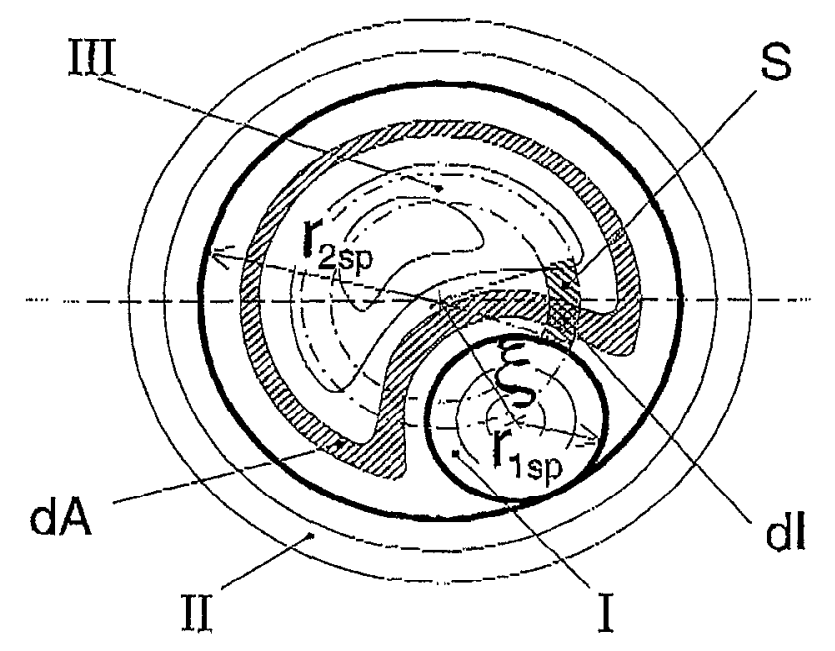

Figure 2: Magnetic island topology; $S$ is the heat source.

Our problem, now, is to specify a function $\psi_{*}(r, \alpha ; t)$ whose contour levels correspond to croissant-shaped surfaces of the type shown in region III of Fig. 2, satisfying the basic reconnection rules. These rules do not define a unique solution for the functional $\psi_{*}$, although they represent a rather strong mathematical constraint. We find it convenient to introduce a Hamiltonian for the magnetic field lines inside the island separatrix (region III of), $H=$ $H\left(\psi_{*}\right)=H\left(r, \alpha_{;} \xi\right)$, such that

$$
H=\frac{\left(r^{2}+\xi^{2}-2 \xi r \cos \alpha-r_{1 s p}^{2}\right)\left(r_{2 s p}^{2}-r^{2}\right)}{r^{2}+\xi^{2}-2 \xi r \cos \alpha}
$$

with $\psi_{* i n}(r)$ and $\xi(t)$ prescribed functions. The island separatrix for a given $\xi$ corresponds to the contour level $H=0$, while $H$ reaches a maximum 
value $H_{\text {max }}(\xi)$, on the island $O$-point Croissant-shaped magnetic surfaces correspord to contour levels $H=: H_{0} \in\left[0, H_{\text {max }}\right]$ Note that the curves $H(r, \alpha ; \xi)=H_{0}$ become circles in both limits $\xi=0$ and $\xi=T_{\text {mix }}$ (in the latter limit, $\left.r_{1 s p}=0\right)$. The function $H=H(A)$ ean be evaluated numerically after computing the area, $A$, pertaining to each $H \doteqdot H_{0}$ surface; from this, the area conservation rule, the function $H \doteq H\left(\psi_{*}\right)$ can be easily constructed. Ontside the island separatrix (regions I and II of Hig. 2) the magnetic surface cross-sections are circular.

Also represented in Fig. 2 is an electron heat source, $S$, localized on the poloidal midplane between radii $r h, r h$ and angles $\theta_{h l}, \theta_{h z}$ Since paraj]el heat conduction is very large, the deposited heat spreads rapidly and uniformily over all flux tubes intersecting the heat deposition region: Then, taking into account plasma rotation (assumed to be rigid for the sake of simplicity), the heated region as sen in the plasma rest frame rotates with angular frequency $\omega_{\text {rot }}$ Thus, in the plasma frame for $S=S(r, \theta, t)$ we have $S=\rho\left[\mathcal{H}\left(r-r_{h x}\right)\left(r_{h z}-r\right)\right] \cdots\left[\mathcal{H}\left(\omega_{\text {rot }}-\theta_{h 1}\right)\left(\theta_{k 2}-\omega_{r o t} t\right)\right]$ where $\mathcal{H}$ is the Heavyside furiction, $\rho=P / V_{h}$ and $V_{h}$ is the deposition yolume Now.; if we denote by d $A$ the cruss-sectional area at a gereric flux tube and by: $\mathrm{d} I$ its intersection with the heated region (Fig. 2); we can write the ECH power density averaged over flux suffaces as $\langle S\rangle(A)=\rho \partial T / \partial A$. Note that the heat is transported radially by parallel heat diffision in a complex magnetic structure such as that of Fig 2 , resulting in an apparently nonlocal heat transport process.

Let us now discuss how the plasnia density and pressure evolve during the growth of the $m=n=1$ island. Let ws distregard, for a moment, the heat source and perpendicular diffusion process. In addition, we neglect magnetic to thermal energy transfer; we have checled that this zccounts for only about $1 \div 2 \%$ of the transferred theat Then the area conservation rule implies the following conservation law for the plasma pressure, $\pi \int_{r_{1} y_{p}}^{r^{2}} p\left(r^{2}\right) d r^{2}=$ $\int_{A} p\left(A^{\prime}\right) \mathrm{d} A^{\prime}$, which in differential form becomes

$$
p(A)=\pi\left[p\left(r_{2 s p}^{2}\right) \frac{\mathrm{d} r_{2 s p}^{2}}{\mathrm{~d} A}-p\left(r_{1 s p}^{2}\right) \frac{\mathrm{d} r_{1 s p}^{2}}{\mathrm{~d} A}\right]=p\left(\psi_{*}\right)
$$

since $A=A\left(\psi_{*}\right)$ through $E q$ (1) The plasma density obeys completely similar relations. We make the simplifying (and experimentally verified [1], [5]) assumption of a tlat electron density. Then, since $p=\pi T, \mathrm{Eq}_{\mathrm{q}}$ (2) car be written for the temperature as well. 
If we now consider the presence of the heat source and the effect of perpendicular heat diffusion, then the pressure and the temperature will depend explicitly on time: $T=T(A, t)$. In this Letter, we assurne $\chi_{\perp}=$ const. It is convenient to specify the heat transport equation in a Lagrangian frame of reference. We also have to take into account the different topology of the three regions I,II,III. Thus, in region III, we obtain the diffusion equation

$$
\frac{3}{2} \frac{\partial T}{\partial t}=\chi_{\perp}\left(\left\langle|\nabla A|^{2}\right\rangle \frac{\partial^{2} T}{\partial A^{2}}+\left\langle\nabla^{2} A\right\rangle \frac{\partial T}{\partial A}\right)+\frac{\langle S\rangle}{n} ;
$$

Similar equations apply to regions I and II, with $A=\pi r^{2}$ and $r$ indicating the distance from the displaced axis in regin $I$. The time evolution of the three regions is specified by the displacement function $\xi(t)$ and by $\psi_{* i n}(r)$, together with Eq. (1). The solution of Eq.(3) requires an initial condition, $T(r, t=0)=T_{i m}(r)$, where at $t=0, \xi(0)=0$, and the following boundary conditions: a condition at the edge of the integration domain, $\tilde{r} \approx 1.5 r_{m i x}$, located in region II, specifically $T(\tilde{r}, t)=T_{i n}(\tilde{r})$; the geometrical conditions $\left(\partial T_{T} / \partial r_{1}\right)_{r_{1}=0}=\left(\partial T_{I I I} / \partial A\right)_{A=0}=0$ on the two magnetic axes of regions I and $M$, respectively. Finally, a condition on the separatrix is required. Note that, with $\chi_{\perp}=0$, a discontinuty of the temperature across the separatrix is in general allowed by (2). With finite $\chi_{\perp}$, a common temperature is achieved approaching the separatrix from any of the three regions $I, I I, I I I$, which is determined by the continuity of the heat flux. Equations (1-3), with the appropriate initial conditions as described above, completely specify our simulation model.

We present a typical solution obtaixzed for the following input parameters [5]: (i) initial helical function $\psi_{* \text { *in }}(x)=\left(\triangle q / q_{0}\right) r_{\text {min }}^{2} x^{2}\left(1-x^{2}\right)$, where $\Delta q=1-q_{0}=1 / 16$, and $x \equiv r / r_{m i x} ;$ (ii) a specified displacement function, $\xi(t)$, inferred from experimental data and shown in Fig.3(a); (iii) a power source $P=180 \mathrm{~kW}, w_{h 1}=0.3$ and $x_{h 2}=0.4$; (iv) a sawtooth period $\tau_{\text {saw }}=2 \mathrm{~ms} ;(v)$ a value $\chi_{\perp}=0.05 \mathrm{~m}^{2} / \mathrm{s}$, (vi) a normalized rotation period, $\tau_{\text {rot }} / \tau_{\text {saw }}=1 / 18$; (viii) an initial temperature profile, $T_{i n}(r)=T_{0}\left(1-r^{3} / a^{2}\right)$, with $T_{0}=0.8 \mathrm{keV}$. Tridimensional reconstructions of the simulated electron temperature at various phases during the growth of the $m=n=1$ magnetic island are shown in Figs. fig3(b), (c). Shown in. Figs. 3(d)-(f) are examples of the simulated temperature profiles. These figures should be compared with the TEXT-U measurements in Fig.1 (see also Figs 10,11 of Ref [5]), as well as the RTP temperature profiles in Figs. 3-4 of [1]. The similarities are quitc 

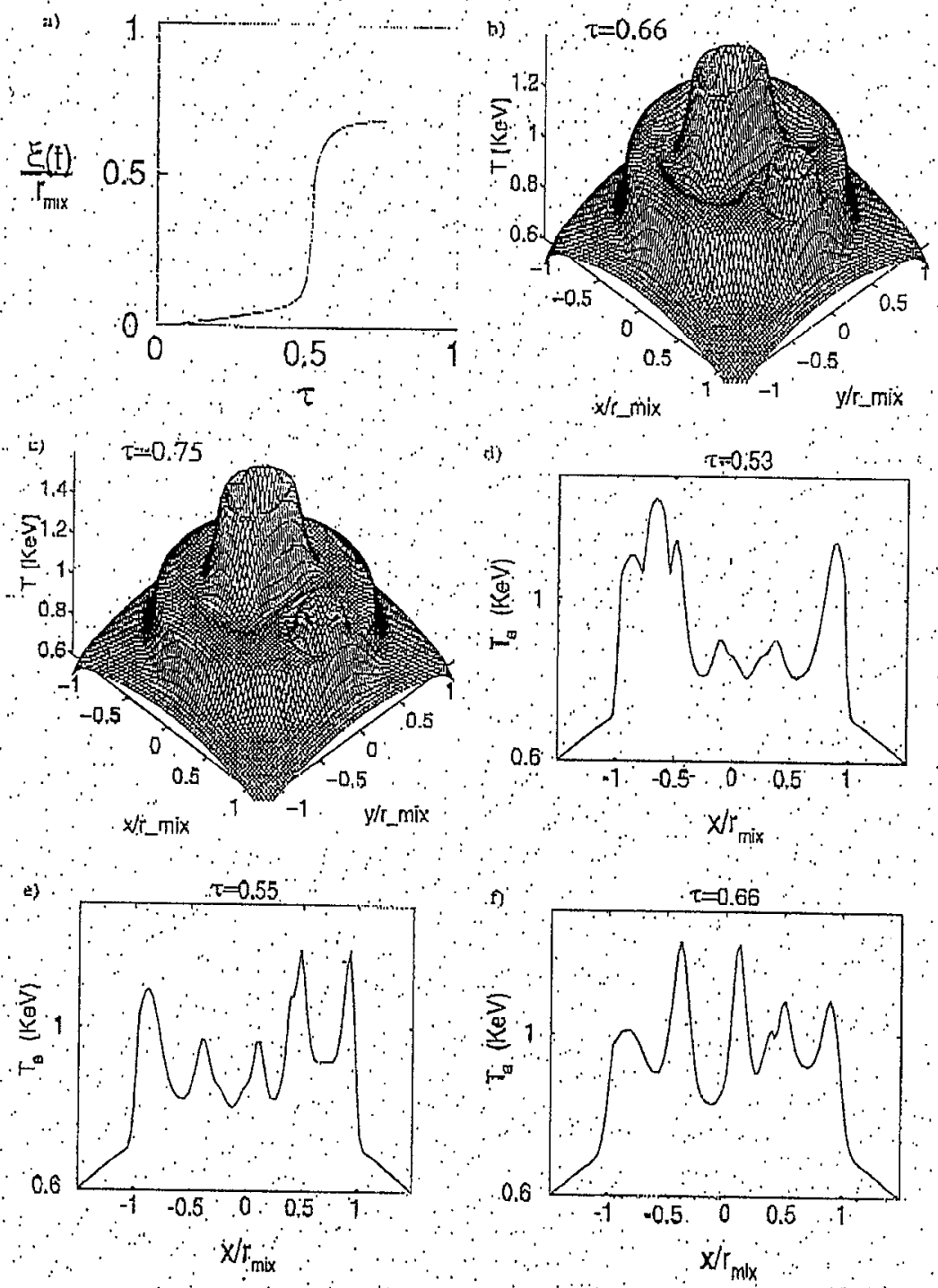

Figure 3: Simulation results (a) displacement furction, $r \equiv\left(t-t_{0}\right) / \tau_{\text {saw }}$ (b), (c): examples of $3=\mathrm{D}$ xeconstructions of $T_{c}$ (d),(e);(f): examples of $T_{c}$ profiles. 
evident. In particular, our model is able to reproduce temperature profiles with four to five peaks, fairly indipendently of the precise size and localization of the heat absorption region. In principle, more peaks are possible with more structured displacement function, $\xi(t)$. In addiction, our model predicts the formation of sharp temperature gradients just outside the mixing radius. This is clear from the 3-D plots in Figs. 3(c,d), where the outer, circular, rim structure corresponds to a region centered on the plot of the island separatrix bordering region II. The rim structure is also quite evident from the TEXT-U experiment reconstiructions shown in Figs, 1(c,d).

We stress that the multi-peaked structure and the sharp gradients are obtained in the simulation even with a constant $\chi_{\perp}$. Indeed, the sharp gradients are a result of heat convection, ratker than diffusion. They are formed as the specific $m=n=1$ resistive internal kink convection partern advects the heat deposited inside the $q=1$ radius to the separatrix region at a rate faster than the heat diffusion rate.

We can think of two possible directions for the refinement of the present model. Firat, one may consider a temperature-dependent diffusion coefficient. If $\chi_{\perp}$ were lower where the temperature is ligher, the peaks in the electron temperature profile would become even sharper. Secondly, one may relax the single helicity assumption for the island evolution. For instance, the $m=2, n=1$ satellite harmonic for the helical flux perturbation would create a band of stochasticity in the field structure centered around the separatrix [10]. Clearly, a fully stochastic magnetic field would not support temperature gradients. All we can say, at the moment, is that judging from the experimental data, this band of stochastic field lines should be relatively narrow during most of the sawtooth ramp.

In conclusion, we have presented a theoretical model that is able to explain the observed multi-peaked temperature profiles, previously referred to as tempetature filarnentation [1], in ECH experiments. In addition, the model predicts the formation of sharp gradients near the sawtooth mixing radius. The model is based on the combined effects of $m=n=1$ island dynamics, localized heat source, very large heat diffusivity along magnetic field lines and plasma rotation. The "transport barrier" just outside the sawtooth mixing radius is in fact a consequence of $m=n=1$ heat convection, rather than r $\theta$ duced perpendicular heat diffusion near the $q=1$ sational surface. We may expect that the sometimes observed minor structures in the electron temperature profile at rational $q$ values can be explained along similar lines, i.e. as 
a consequence of macroscopic island dynamics with $m=q_{\text {ration }}$

The authors wish to acknowledge useftl discussion with Drs. N.J. Lopez Cardozo, A. Pochelon and A. Pyetrzyk. Ore of us (F.P. was supported in part by the Italiar Research Conncil (C.N.R.), while A.W ackiowledges the Texas Atomic Energy Research Foundation for funarial support.

\section{References}

[1] N.: J Lopez Cardozo et al., Phys Rev Lett., 73, 256, (1994):

[2] M. R. de Baris et al, Phys Rev Lett, $78 ; 4573,(1997)$.

[3] No.J Lopez Cardozo' et cil, Plasma Phys. Contr. Fusion, 39, B303, (1997)

[4] von Goeler et al:, Phys. Rev. Lett, $\mathbf{3 3}, 1201,(1973)$ :

[5] G. Cima et al., Plasma Phys Contr Fusion, 40, 1149, (1998)

[6] Z. A Pielrayk el al, subxhted to Nucl. Fusion, (1998):

[7] V Erckmann and U. Gasparino, Plasma Phys Contr, Fusion, 36, 1869, (1998).

[8] B. Coppi et al, Fiz: Plasmy, 2,961, (1976); [Sov J Plasma Phys 2 $533,(1976)]$ :

[9] B. B. Kadomtsev, Fiz. Plasmy, 1,710 , (1975); [Sov, J Plasma Phys, I, $389,(19,75)]$

[10] A J Lichtenberg, Nucl. Fusion, 24; 1277, (1984)

This work was performed under the auspices of the U.S DOE by LLNL under contract No. W-7405-Eng-48. 